\title{
Article \\ New Results on Fourth-Order Differential Subordination and Superordination for Univalent Analytic Functions Involving a Linear Operator
}

\author{
Bassim Kareem Mihsin ${ }^{1}{ }^{*}$, Waggas Galib Atshan ${ }^{2} \mathbb{D}$, Shatha S. Alhily ${ }^{1}$ and Alina Alb Lupaş ${ }^{3} \mathbb{D}$ \\ 1 Department of Mathematics, College of Science, Mustansiriyah University, Baghdad 10052, Iraq; \\ shathamaths@yahoo.co.uk or shathamaths@omstansiriyah.edu.iq \\ 2 Department of Mathematics, College of Science, University Al-Qadisiyah, Diwaniyah 58001, Iraq; \\ waggashnd@gmail.com or waggas.galib@qu.edu.iq \\ 3 Department of Mathematics and Computer Science, University of Oradea, 410087 Oradea, Romania; \\ alblupas@gmail.com \\ * Correspondence: basmk3756@gmail.com
}

Citation: Mihsin, B.K.; Atshan, W.G.; Alhily, S.S.; Lupaş, A.A. New Results on Fourth-Order Differential Subordination and Superordination for Univalent Analytic Functions Involving a Linear Operator. Symmetry 2022, 14, 324. https:// doi.org/10.3390/sym14020324

Academic Editor: Ioan Rașa

Received: 10 December 2021

Accepted: 10 January 2022

Published: 5 February 2022

Publisher's Note: MDPI stays neutral with regard to jurisdictional claims in published maps and institutional affiliations.

Copyright: () 2022 by the authors Licensee MDPI, Basel, Switzerland. This article is an open access article distributed under the terms and conditions of the Creative Commons Attribution (CC BY) license (https:// creativecommons.org/licenses/by/ $4.0 /)$.

\begin{abstract}
We present several new results for fourth-order differential subordination and superordination in this paper by using the differential linear operator $\Gamma_{\pi, \rho, \beta, \mu} f(z)$. Relevant connections between the new results presented here and those considered in previous works are addressed. The properties and results concerning the differential subordination theory are symmetric to the properties obtained using the differential superordination theory, and by combining them, sandwich-type theorems are obtained.
\end{abstract}

Keywords: fourth-order differential subordination; admissible function; Hadamard product; differential subordination; superordination; sandwich theorem; dominant; subordinate

MSC Classification: 30C45

\section{Introduction}

The investigation conducted in this paper uses the well-known concepts of differential subordination and differential superordination. The concept of differential subordination introduced by Miller and Mocanu is presented in the monograph published in 2000 [1], and the concept of differential superordination was introduced by the same authors as dual concept to subordination in 2003 [2]. Third-order differential inequalities in the complex plane were considered in 1992 [3], and the concept of third-order differential subordination was introduced in 2011 by Antonino and Miller [4]. Further investigations were done on third-order differential subordination results for univalent analytic functions involving an operator in 2020 [5], and continuing the idea, the concept of fourth-order differential subordination was introduced and studied in $2020[6,7]$. Further results were published in 2021 [8] regarding the new concepts of higher-order differential subordinations. The present paper continues this study.

Interesting results were recently obtained regarding higher order differential subordination involving an operator [9-12], and other interesting results involving operators emerged as can be seen in papers published in 2020 [13-16] and 2021 [17-19]. These results motivated the introduction of the new operator, which will be presented at the end of this first section in Definition 1, and will be used in the next sections to obtain the original results regarding fourth-order differential subordinations and superordinations.

The usual environment provides the context for the present investigation. Well-known notations and definitions used for obtaining the original results are next presented. 
$\mathcal{K}\left(\mathrm{U}^{\circ}\right)$ denotes the family of analytic functions in $\mathrm{U}^{\circ}$ that have the form:

$$
\begin{gathered}
\mathcal{K}[\mathrm{a}, \mathfrak{n}]=\left\{f \in \mathcal{K}\left(\mathrm{U}^{\circ}\right): f(z)=a+a_{\mathfrak{n}} z^{\mathfrak{n}}+a_{\mathfrak{n}+1} z^{\mathfrak{n}+1}+\cdots\right\}, \\
a \in \mathbb{C}, \mathfrak{n} \in N=\{1,2, \ldots\},
\end{gathered}
$$

and let $\mho_{\mathfrak{n}}$ be the collection of the form:

$$
\mho_{\mathfrak{n}}=\left\{f \in \mathcal{K}\left(U^{\circ}\right): f(z)=z+a_{\mathfrak{n}+1} z^{\mathfrak{n}+1}+\cdots\right\},
$$

where $\mho_{1}=\mho$, the subclass of normalized analytic functions in $\mathrm{U}^{\circ}$. Further, indicate by $\mathrm{M}$ the subfamily of $\mathcal{K}\left(\mathrm{U}^{\circ}\right)$ of the form:

$$
f(z)=z+\sum_{\mathfrak{n}=2}^{\infty} a_{\mathfrak{n}} z^{\mathfrak{n}}, z \in \mathrm{U}^{\circ},
$$

which are univalent in $\mathrm{U}^{\circ}$. For analytic functions $f$ and $F$, the function $f$ is said to be subordinate to $F$, if

$$
f(z)=F(\Theta(z)),\left(z \in U^{\circ}\right),
$$

where $\Theta(z)$ is analytic and $\Theta(0)=0,|\Theta(z)|<1$. This subordination is indicated by $f(z) \prec F(z)$.

Cho and Kim [20] proposed the multiplier transformation as a linear operator. Let $\mathfrak{n}$ be any integer; the multiplier transformation $\mathcal{L}_{\mu}^{\beta}: M \rightarrow M$ is given by $\mathcal{L}_{\mu}^{\beta} f(z)=z+$ $\sum_{\mathfrak{n}=2}^{\infty}\left(\frac{\mathfrak{n}+\mu}{1+\mu}\right)^{\beta} a_{\mathfrak{n}} z^{\mathfrak{n}}, \mu \geq 0, \beta \in \mathbb{Z}=\{\cdots,-1,0,1, \cdots\}$.

The Hurwitz-Lerch Zeta function [21] is

$$
\begin{gathered}
\zeta_{\pi, \rho}(z)=\frac{1}{\rho^{\pi}}+\sum_{\mathfrak{n}=1}^{\infty} \frac{z^{\mathfrak{n}}}{(\mathfrak{n}+\rho)^{\pi}}, \\
\left(\rho \in \mathbb{C} \backslash Z_{o}^{-}=\{0,-1,-2, \ldots\}, \pi \in \mathbb{C}, \text { where }|z|<1, \mathcal{R} e(\pi)>1, z \in \partial U^{\circ}\right)
\end{gathered}
$$

By making use of the following normalized function, we have:

$$
G_{\pi, \rho}(z)=(1+\rho)^{\pi}\left[\zeta_{\pi, \rho}(z)-\rho^{-\pi}\right]=z+\sum_{\mathfrak{n}=2}^{\infty}\left(\frac{1+\rho}{\mathfrak{n}+\rho}\right)^{\pi}, z \in U^{\circ} .
$$

If $f, g \in M$, where $f$ given by (1) and $g$ is defined by

$$
g(z)=z+\sum_{\mathfrak{n}=2}^{\infty} b_{\mathfrak{n}} z^{\mathfrak{n}}, z \in \mathrm{U}^{\circ},
$$

then

$$
(f * g)(z)=z+\sum_{\mathfrak{n}=2}^{\infty} a_{\mathfrak{n}} b_{\mathfrak{n}} z^{\mathfrak{n}}=(g * f)(z) .
$$

Using the convolution defined above, a new operator is next introduced as the original part of the present paper.

Definition 1. Assume $f \in M, z \in \partial U^{\circ}, \rho \in \mathbb{C} \backslash Z_{o}^{-}=\{0,-1,-2, \ldots\}$, where $|z|<1$, $\mathcal{R} e(\pi)>1, \mu \geq 0, \beta \in \mathbb{Z}, \pi \in \mathbb{C}$; we define new operator $\Gamma_{\pi, \rho, \beta, \mu} f(z): M \rightarrow M$, where

$$
\Gamma_{\pi, \rho, \beta, \mu} f(z)=G_{\pi, \rho(z)} * \mathcal{L}_{\mu}^{\beta} f(z)=z+\sum_{\mathfrak{n}=2}^{\infty}\left(\frac{1+\rho}{\mathfrak{n}+\rho}\right)^{\pi}\left(\frac{\mathfrak{n}+\mu}{1+\mu}\right)^{\beta} a_{\mathfrak{n}} z^{\mathfrak{n}} .
$$


After a simple computation, we obtain the relation:

$$
z\left(\Gamma_{\pi, \rho, \beta, \mu} f(z)\right)^{\prime}=(1+\mu) \Gamma_{\pi, \rho, \beta+1, \mu} f(z)-\mu \Gamma_{\pi, \rho, \beta, \mu} f(z) .
$$

\section{Problem Formulation}

The subcollection of various analytic and univalent functions, which are connected to differential subordination and superordination in the open unit disk $\mathrm{U}^{\circ}$, has been initiated in recent times from a variety of intriguing outcomes and perspectives (cf. [7,22-28]). Additionally, several authors obtained good results on second- and third-order differential subordination; e.g., [29-35].

In order to demonstrate the original results, we will need the basic concepts of fourthorder theory previously introduced, which we present below showing the papers where they first appeared.

Definition 2. Ref. [4]: Assume that $\mathbb{Q}$ is called the set of functions $\mathbb{q}$ that are univalent and analytic on the set $\bar{U}^{\circ} \backslash E(\mathbb{q})$, where $E(\mathbb{q})=\left\{\mathcal{J}: \mathcal{J} \in \partial U^{\circ}\right.$ and $\left.\lim _{z \rightarrow \mathcal{J}} \mathbb{q}(z)=\infty\right\}$ are such that $\min \left|\mathbb{q}^{\prime}(\mathcal{J})\right|=\gamma>0$ for $\mathcal{J} \in \partial U^{\circ} \backslash E(\mathbb{q})$. In addition, indicate by $\mathbb{Q}(a)$ the subclass of function $\mathbb{q}$ for which $\mathbb{\mathbb { }}(0)=a$. Note that $\mathbb{Q}_{1}=\mathbb{Q}(1)=\{\mathbb{Q}(z) \in \mathbb{Q}: \mathbb{q}(0)=1\}$.

Definition 3. See [6,7]: Assume that $k$ is univalent in $U^{\circ}$ and $\psi: \mathbb{C}^{5} \times U^{\circ} \rightarrow \mathbb{C}$. If the analytic function $p$ fulfills the fourth-order differential subordination

$$
\psi\left(p(z), z p^{\prime}(z), z^{2} p^{\prime \prime}(z), z^{3} p^{\prime \prime \prime}(z), z^{4} p^{\prime \prime \prime \prime}(z) ; z\right) \prec k(z),
$$

then the function $p$ is named a solution of the differential subordination (4). A univalent function $\mathbb{q}$ is named a dominant of the solutions of the differential subordination if $p \prec \mathbb{q}$ for all $p$ satisfying (4). A dominant $\widetilde{\mathbb{q}}(z)$ that fulfills $\widetilde{\mathbb{q}} \prec \mathbb{q}$ for all dominants $\mathbb{q}$ of (4) is named the best dominant.

Definition 4. See [6,7]: Assume that $\mathbb{q} \in \mathbb{Q}$ and $\Omega$ is a set in $\mathbb{C}$. The admissible functions class $\Phi_{\mathfrak{n}}[\Omega, \mathbb{q}],(\mathfrak{n} \in N \backslash\{2\})$ consists of those functions $\psi: \mathbb{C}^{5} \times U^{\circ} \rightarrow \mathbb{C}$ that fulfill the following admissibility condition:

$$
\psi(\mathfrak{r}, \mathfrak{s}, \mathfrak{t}, \mathfrak{u}, b ; z) \notin \Omega,
$$

wherever

$$
\begin{array}{r}
\mathfrak{r}=\mathfrak{q}(\tau), \mathfrak{s}=\mathfrak{m} \tau \mathbb{q}^{\prime}(\tau), \quad \mathcal{R} e\left(\frac{\mathfrak{t}}{\mathfrak{s}}+1\right) \geq \mathfrak{m} \mathcal{R} e\left(1+\frac{\tau \mathbb{q}^{\prime \prime}(\tau)}{\mathbb{q}^{\prime}(\tau)}\right), \\
e \mathcal{R}\left(\frac{\mathfrak{u}}{\mathfrak{s}}\right) \geq \mathfrak{m}^{2} \mathcal{R} e\left(\frac{\tau^{2} \mathbb{q}^{\prime \prime \prime}(\tau)}{\mathbb{q}^{\prime}(\tau)}\right), e \mathcal{R}\left(\frac{b}{s}\right) \geq \mathfrak{m}^{3} \mathcal{R} e\left(\frac{\tau^{3} \mathfrak{q}^{\prime \prime \prime \prime \prime}(\tau)}{\mathbb{q}^{\prime}(\tau)}\right), \\
\left(z \in U^{\circ}, \tau \in \partial U^{\circ} \backslash \mathrm{E}(\mathbb{q}) \text { and } \mathfrak{m} \geq \mathfrak{n}\right) .
\end{array}
$$

Theorem 1. See [7]: Let $p \in \mathcal{K}[a, \mathfrak{n}],(\mathfrak{n} \in N \backslash\{2\})$. In addition, let $\mathbb{q} \in \mathbb{Q}$ and fulfill the conditions:

$$
\mathcal{R} e\left(\frac{\tau^{2} \mathbb{q}^{\prime \prime \prime}(\tau)}{\mathbb{q}^{\prime}(\tau)}\right) \geq 0, \quad\left|\left(\frac{z^{2} p^{\prime \prime}(\tau)}{\mathbb{q}^{\prime}(\tau)}\right)\right| \leq \mathfrak{m}^{2},
$$

where $z \in U^{\circ}, \tau \in \partial U^{\circ} \backslash E(\mathbb{q})$ and $\mathfrak{m} \geq \mathfrak{n}$. If $\psi \in \Phi_{\mathfrak{n}}[\Omega, \mathbb{q}], \Omega$ is a set in $\mathbb{C}$ and $\psi\left(p(\boldsymbol{z}), z p^{\prime}(\boldsymbol{z}), z^{2} p^{\prime \prime}(\boldsymbol{z}), z^{3} p^{\prime \prime \prime}(\boldsymbol{z}), z^{4} p^{\prime \prime \prime \prime}(\boldsymbol{z}) ; \boldsymbol{z}\right) \in \Omega$, then $p(\boldsymbol{z}) \prec \mathbb{q}(\boldsymbol{z}), \quad z \in U^{\circ}$.

Definition 5. See [6,7]: Assume that $\psi: \mathbb{C}^{5} \times U^{\circ} \rightarrow \mathbb{C}$ and $k$ is an analytic function in $U^{\circ}$. If $p(z)$ and

$$
\psi\left(p(z), z p^{\prime}(z), z^{2} p^{\prime \prime}(z), z^{3} p^{\prime \prime \prime}(z), z^{4} p^{\prime \prime \prime \prime}(z) ; z\right)
$$


are univalent in $U^{\circ}$ and satisfy the fourth-order differential superordination

$$
k(z) \prec \psi\left(p(z), z p^{\prime}(z), z^{2} p^{\prime \prime}(z), z^{3} p^{\prime \prime \prime}(z), z^{4} p^{\prime \prime \prime \prime}(z) ; z\right),
$$

then $p(z)$ is called a solution of the differential superordination. An analytic function $\mathbb{q}(z)$ is denoted a subordinate of the solutions of the differential superordination or more simply a subordinate if $\mathbb{q}(z) \prec p(z)$ for all $p(z)$ satisfying (6). A univalent subordinate $\widetilde{\mathbb{q}}(z)$ that satisfies the condition $\mathbb{q}(z) \prec \widetilde{\mathbb{q}}(z)$ for all subordinates $\mathbb{q}(z)$ of $(6)$ is referred to as the best subordinate. We note that the best subordinate is unique up to a rotation of $U^{\circ}$.

Definition 6. See [6,7]: Assume $\mathbb{q}(z) \in \mathcal{K}[a, \mathfrak{n}], \mathbb{q}^{\prime}(z) \neq 0$ and $\Omega$ is a set in $\mathbb{C}$. The class of admissible functions $\Phi_{\mathfrak{n}}^{\prime}[\Omega, \mathbb{q}]$ consists of those functions:

$$
\psi: \mathbb{C}^{5} \times \bar{U}^{\circ} \rightarrow \mathbb{C}
$$

that satisfy the following admissibility condition:

$$
\psi(\mathfrak{r}, \mathfrak{s}, \mathfrak{t}, \mathfrak{u}, b ; z) \notin \Omega,
$$

wherever

$$
\mathfrak{r}=\mathbb{q}(z), \mathfrak{s}=\frac{1}{\kappa} z_{\mathbb{q}^{\prime}}(\tau), \quad \mathcal{R} e\left(\frac{\mathfrak{t}}{\mathfrak{s}}+1\right) \geq \frac{1}{\kappa} \mathcal{R} e\left(\frac{z_{\mathbb{q}^{\prime \prime}}(z)}{\mathbb{q}^{\prime}(z)}+1\right)
$$

and

$$
e \mathcal{R}\left(\frac{\mathfrak{u}}{\mathfrak{s}}\right) \geq \frac{1}{\kappa^{2}} \mathcal{R} e\left(\frac{\tau^{2} \mathbb{q}^{\prime \prime \prime}(z)}{\mathbb{q}^{\prime}(z)}\right), e \mathcal{R}\left(\frac{\mathfrak{t}}{\mathfrak{s}}\right) \geq \frac{1}{\kappa^{3}} \mathcal{R} e\left(\frac{\tau^{3} \mathbb{q}^{\prime \prime \prime \prime}(z)}{\mathbb{q}^{\prime}(z)}\right),
$$

where $\tau \in \partial U^{\circ}, z \in U^{\circ}$ and $\kappa \geq \mathfrak{n} \geq 3$.

Theorem 2. See $[6,7]:$ Assume that $\psi \in \Phi_{\mathfrak{n}}^{\prime}[\Omega, \mathbb{q}]$ and $\mathbb{q}(z) \in \mathcal{K}[a, \mathfrak{n}]$. If

$$
\psi\left(p(z), z p^{\prime}(z), z^{2} p^{\prime \prime}(z), z^{3} p^{\prime \prime \prime}(z), z^{4} p^{\prime \prime \prime \prime}(z) ; z\right)
$$

is univalent in $U^{\circ}$ and $p(z) \in \boldsymbol{Q}(a)$ satisfy the conditions

$$
\mathcal{R} e\left(\frac{z^{2} \mathbb{q}^{\prime \prime \prime}(z)}{\mathbb{q}^{\prime}(z)}\right) \geq 0, \quad\left|\left(\frac{z^{2} \mathbb{q}^{\prime \prime}(z)}{\mathbb{q}^{\prime}(z)}\right)\right| \leq \frac{1}{\kappa^{2^{\prime \prime}}}
$$

$z \in U^{\circ}, \tau \in \partial U^{\circ}$, and $\kappa \geq \mathfrak{n} \geq 3$, then where

$$
\Omega \subset\left\{\left(\psi\left(p(z), z p^{\prime}(z), z^{2} p^{\prime \prime}(z), z^{3} p^{\prime \prime \prime}(z), z^{4} p^{\prime \prime \prime \prime}(z) ; z\right), z \in U^{\circ}\right)\right\}
$$

thus, $\mathbb{q}(z) \prec p(z), z \in U^{\circ}$.

Using those known definitions and results, in the next two sections, we prove new fourth-order differential subordination and superordination results involving the operator introduced in Definition 1. Further, in the last section of the paper, we combine the results for obtaining a sandwich-type theorem.

\section{Fourth-Order Differential Subordination Results Using the Operator $\Gamma_{\pi, \rho, \beta, \mu} f(z)$}

We give the class of admissible functions, which is required in proving differential subordination theorems using the operator $\Gamma_{\pi, \rho, \beta, \mu} f(z)$ given by (2). 
Definition 7. Assume $\mathbb{q} \in \boldsymbol{Q}_{1} \cap \mathcal{K}_{1}$ and $\Omega$ is a set in $\mathbb{C}$. Let $\theta_{\Gamma}[\Omega, \mathbb{q}]$ be the class of admissible functions that consists of those functions $Y: \mathbb{C}^{5} \times U^{\circ} \rightarrow \mathbb{C}$ that satisfy the following admissibility condition:

$$
Y(\mathrm{r}, \mathbb{s}, \mathrm{x}, \mathrm{y}, \mathrm{g}, z) \notin \Omega,
$$

wherever

$$
\begin{gathered}
\mathbb{r}=\mathbb{q}(\mathcal{J}), \quad \mathbb{s}=\frac{\mathfrak{m} \mathcal{J} \mathbb{q}^{\prime}(\tau \mathcal{J})+\mu \mathbb{q}(z)}{1+\mu}, \\
\mathcal{R e}\left\{\frac{(1+\mu)^{2} \mathbb{x}-\mu^{2} \mathbb{r}}{(1+\mu) \mathbb{s}-\mu \mathrm{r}}-2 \mu\right\} \geq \mathfrak{m} \mathcal{R e}\left\{\frac{\mathcal{J} \mathbb{q}^{\prime \prime}(\mathcal{J})}{\mathfrak{q}^{\prime}(\mathcal{J})}+1\right\}, \\
\mathcal{R e}\left\{\frac{(1+\mu)^{2}[(1+\mu) \mathbb{y}-(3+3 \mu) \mathbb{x}]+\left(3 \mu^{2}+2 \mu^{3}\right) \mathbb{r}}{(1+\mu) \mathbb{s}-\mu \mathrm{r}}+\left(2+6 \mu+3 \mu^{2}\right)\right\} \geq \mathfrak{m}^{2} \mathcal{R e}\left\{\frac{\mathcal{J}^{2} \mathbb{q}^{\prime \prime \prime}(\mathcal{J})}{\mathbb{q}^{\prime}(\mathcal{J})}\right\},
\end{gathered}
$$

and

$$
\begin{aligned}
\mathcal{R} e\left\{\frac{(1+\mu)\left[(1+\mu)^{3} \mathrm{~g}-(1+\mu)^{2}(6+4 \mu) \mathrm{y}+(1+\mu)\left(11+18 \mu+8 \mu^{2}\right) \mathbb{x}\right.}{(1+\mu) \mathbb{s}+\mu \mathrm{r}}\right. \\
\left.\frac{-\left(6 \mathbb{s}+22 \mu+18 \mu^{2}+8 \mu^{3}\right]+\left(6 \mu+11 \mu^{2}+6 \mu^{3}+3 \mu^{4}\right) \mathbb{s}}{(1+\mu) \mathbb{s}+\mu \mathrm{r}}\right\} \geq \mathfrak{m}^{3} \mathcal{R} e\left\{\frac{\mathcal{J}^{3} \mathbb{q}^{\prime \prime \prime \prime}(\mathcal{J})}{\mathbb{q}^{\prime}(\mathcal{J})}\right\},
\end{aligned}
$$

where $z \in U^{\circ}, \mu \in \partial U^{\circ} \backslash E(\mathbb{q}), \mu>-1$ and $\mathfrak{m} \geq 3$.

Theorem 3. Assume that $Y \in \theta_{\Gamma}[\Omega, \mathbb{q}]$. If $f \in \mho$ and $\mathbb{q} \in \mathbb{Q}_{1}$ satisfy the following conditions:

$$
\mathcal{R} e\left(\frac{\mathcal{J}^{2} \mathbb{q}^{\prime \prime \prime}(\mathcal{J})}{\mathbb{q}^{\prime}(\mathcal{J})}\right) \geq 0, \quad\left|\left(\frac{\Gamma_{\pi, \rho, \beta, \mu} f(\boldsymbol{z})}{\mathbb{q}^{\prime}(\mathcal{J})}\right)\right| \leq \mathfrak{m}^{2}
$$

and

$$
\begin{gathered}
\left\{\mathrm{Y}\left(\Gamma_{\pi, \rho, \beta, \mu} f(z), \Gamma_{\pi, \rho, \beta+1, \mu} f(z), \Gamma_{\pi, \rho, \beta+2, \mu} f(z), \Gamma_{\pi, \rho, \beta+3, \mu} f(z), \Gamma_{\pi, \rho, \beta+4, \mu} f(z)\right): z \in U^{\circ}\right\} \subset \Omega, \\
\text { then } \Gamma_{\pi, \rho, \beta, \mu} f(z) \prec \mathbb{q}(z), z \in U^{\circ} .
\end{gathered}
$$

\section{Proof.}

$$
\text { Put } \mathrm{p}(z)=\Gamma_{\pi, \rho, \beta, \mu} \mathrm{f}(z)
$$

Now, by differentiating (9) with respect to $z$ and by applying (3), we obtain:

$$
\Gamma_{\pi, \rho, \beta+1, \mu} f(z)=\frac{z p^{\prime}(\boldsymbol{z})+\mu p(z)}{(1+\mu)} .
$$

Further computations show that

$$
\begin{gathered}
\Gamma_{\pi, \rho, \beta+2, \mu} f(z)=\frac{z^{2} p^{\prime \prime}(z)+(12 \mu+1) z p^{\prime}(z)+\mu^{2} p(z)}{(1+\mu)^{2}}, \\
\Gamma_{\pi, \rho, \beta+3, \mu} f(z)=\frac{\mu^{3} p^{\prime \prime \prime}(z)+(3+3 \mu) z^{2} p^{\prime \prime}(z)+\left(1+3 \mu+3 \mu^{2}\right) z p^{\prime}(z)+\mu^{3} p(z)}{(1+\mu)^{4}}
\end{gathered}
$$

and

$$
\begin{aligned}
& \Gamma_{\pi, \rho, \beta+4, \mu} f(\boldsymbol{z}) \\
& =\frac{\boldsymbol{z}^{4} p^{\prime \prime \prime \prime}(\boldsymbol{z})+(6+4 \mu) \boldsymbol{z}^{3} p^{\prime \prime \prime}(\boldsymbol{z})+\left(7+12 \mu+4 \mu^{2}\right) \boldsymbol{z}^{2} p^{\prime \prime}(\boldsymbol{z})+\left(1+4 \mu+4 \mu^{2}+4 \mu^{3}\right) z p^{\prime}(\boldsymbol{z})+\mu^{4} p(\boldsymbol{z})}{(1+\mu)^{4}} .
\end{aligned}
$$


Now, we present the transformation from $\mathbb{C}^{5}$ to $\mathbb{C}$ by

$$
\begin{gathered}
\mathbb{r}(\mathfrak{r}, \mathfrak{s}, \mathfrak{t}, \mathfrak{u}, b ; z)=\mathfrak{r}, \mathbb{s}(\mathfrak{r}, \mathfrak{s}, \mathfrak{t}, \mathfrak{u}, b ; z)=\frac{\mathfrak{s}+\mu \mathfrak{r}}{1+\mu}, \\
\mathbb{x}(\mathfrak{r}, \mathfrak{s}, \mathfrak{t}, \mathfrak{u}, b ; z)=\frac{\mathfrak{t}+(1+2 \mu) \mathfrak{s}+\mu^{2} \mathfrak{r}}{(1+\mu)^{2}}, \\
\mathbb{y}(\mathfrak{r}, \mathfrak{s}, \mathfrak{t}, \mathfrak{u}, b ; z)=\frac{\mathfrak{u}+(3+3 \mu) \mathfrak{t}+\left(1+3 \mu+3 \mu^{2}\right) \mathfrak{s}+\mu^{3} \mathfrak{r}}{(1+\mu)^{3}},
\end{gathered}
$$

and

$$
\mathrm{g}(\mathfrak{r}, \mathfrak{s}, \mathfrak{t}, \mathfrak{u}, b ; z)=\frac{b+(4 \mu+6) \mathfrak{u}+\left(4 \mu^{2}+12 \mu+7\right) \mathfrak{t}+\left(4 \mu^{3}+4 \mu^{2}+4 \mu+1\right) \mathfrak{s}+\mu^{4} \mathfrak{r}}{(1+\mu)^{4}} .
$$

Assume

$$
\begin{gathered}
\psi(\mathfrak{r}, \mathfrak{s}, \mathfrak{t}, \mathfrak{u}, b ; z)=\mathrm{Y}(\mathfrak{r}, \mathbb{\mathbb { S }}, \mathbb{\mathbb { X }}, \mathbb{\mathbb { V }}, \mathbb{q}, z), \\
=\mathrm{Y}\left(\mathfrak{r}, \frac{\mathfrak{s}+\mu \mathfrak{r}}{1+\mu}, \frac{\mathfrak{t}+(1+2 \mu) \mathfrak{s}+\mu^{2} \mathfrak{r}}{(1+\mu)^{2}}, \frac{\mathfrak{u}+(3+3 \mu) \mathfrak{t}+\left(1+3 \mu+3 \mu^{2}\right) \mathfrak{s}+\mu^{3} \mathfrak{r}}{(1+\mu)^{3}},\right. \\
\left.\frac{b+(6+4 \mu) \mathfrak{u}+\left(7+12 \mu+4 \mu^{2}\right) \mathfrak{t}+\left(1+4 \mu+4 \mu^{2}+4 \mu^{3}\right) \mathfrak{s}+\mu^{4} \mathfrak{r}}{(1+\mu)^{4}} ; z\right) .
\end{gathered}
$$

We conclude the proof by using Theorem 1, and by using Equation (9) in (13), we obtain from (15) that

$$
\begin{gathered}
\left(\psi\left(p(z), z p^{\prime}(z), z^{2} p^{\prime \prime}(z), z^{3} p^{\prime \prime \prime}(z), z^{4} p^{\prime \prime \prime \prime}(z) ; z\right)=\right. \\
Y\left(\Gamma_{\pi, \rho, \beta, \mu} f(z), \Gamma_{\pi, \rho, \beta+1, \mu} f(z), \Gamma_{\pi, \rho, \beta+2, \mu} f(z), \Gamma_{\pi, \rho, \beta+3, \mu} f(z), \Gamma_{\pi, \rho, \beta+4, \mu} f(z) ; z\right) .
\end{gathered}
$$

Therefore, (8) transforms into

$$
\psi\left(p(z), z p^{\prime}(z), z^{2} p^{\prime \prime}(z), z^{3} p^{\prime \prime \prime}(z), z^{4} p^{\prime \prime \prime \prime}(z) ; z\right) \in \Omega,
$$

and we observe that

$$
\begin{gathered}
\frac{\mathfrak{t}}{\mathfrak{s}}+1=\frac{(1+\mu)^{2} \mathbb{x}-\mu^{2} \mathbb{r}}{(1+\mu) \mathbb{s}-\mu \mathrm{r}}-2 \mu, \\
\frac{\mathfrak{u}}{\mathfrak{s}}=\frac{(1+\mu)^{2}[(1+\mu) \mathbb{y}-(3+3 \mu) \mathbb{x}]+\left(3 \mu^{2}+2 \mu^{3}\right) \mathbb{r}}{(1+\mu) \mathbb{s}-\mu \mathrm{r}}+\left(2+6 \mu+3 \mu^{2}\right),
\end{gathered}
$$

and

$$
\frac{b}{\mathfrak{s}}=\frac{(1+\mu)\left[(1+\mu)^{3} \mathrm{~g}-(1+\mu)^{2}(6+4 \mu) \mathrm{y}+(1+\mu)\left(11+18 \mu+8 \mu^{2}\right) \mathbb{X}-\left(6+22 \mu+18 \mu^{2}+8 \mu^{3}\right) \mathbb{s}\right]+\left(6 \mu+11 \mu^{2}+6 \mu^{3}+3 \mu^{4}\right) \mathrm{r}}{(1+\mu) \mathbb{S}-\mu \mathrm{r}} .
$$

Hence, we have the equivalent of the admissibility condition for $\theta_{\Gamma}[\Omega, \mathbb{q}]$ in Definition 7 with the admissibility condition for $\psi \in \Phi_{\mathfrak{n}}[\Omega, \mathbb{q}]$ as known in Definition $4, \mathfrak{n}=3$. Thus, by using Theorem 1 with Equation (7), we have $\mathrm{p}(z)=\Gamma_{\pi, \rho, \beta, \mu} f(z) \prec \mathbb{q}(z)$.

The below corollary is the extension of the above theorem for the case where the action of $q(z)$ on $\partial U^{\circ}$ is unknown. 
Corollary 1. Assume the function $\mathbb{q}(z)$ is univalent in $U^{\circ}$ with $\mathbb{q}(0)=1$ and $\Omega \subset \mathbb{C}$. Assume $Y \in \theta_{\Gamma}\left[\Omega, \mathbb{q}_{\gamma}\right]$ for some $\gamma \in(0,1)$, such that $\mathbb{q}_{\gamma}(z)=\mathbb{q}(\gamma \boldsymbol{z})$. If the function $f(z) \in \mho$ and $\mathbb{A}_{\gamma}(z)$ satisfy the following conditions:

$$
\mathcal{R} e\left(\frac{\mathcal{J}^{2} \mathbb{q}^{\prime \prime \prime}(z)}{\mathbb{q}^{\prime}(z)}\right) \geq 0,\left|\left(\frac{\Gamma_{\pi, \rho, \beta+2, \mu} f(z)}{\mathbb{q}^{\prime}(z)}\right)\right| \leq \mathfrak{m}^{2},\left(z \in U^{\circ}, \mathcal{J} \in \partial U^{\circ} \backslash E\left(\mathbb{q}_{\gamma}\right)\right)
$$

and

$\left\{\mathrm{Y}\left(\Gamma_{\pi, \rho, \beta, \mu} f(z), \Gamma_{\pi, \rho, \beta+1, \mu} f(z), \Gamma_{\pi, \rho, \beta+2, \mu} f(z), \Gamma_{\pi, \rho, \beta+3, \mu} f(z), \Gamma_{\pi, \rho, \beta+4, \mu} f(z)\right): z \in U^{\circ}\right\} \subset \Omega$.

Then

$$
\Gamma_{\pi, \rho, \beta, \mu} f(z) \prec \mathbb{q}(z), z \in U^{\circ}
$$

Proof. By applying the theorem above, we have $\Gamma_{\pi, \rho, \beta, \mu} \mathrm{f}(z) \prec \mathbb{q}_{\gamma}(z)$. Then, we have the result from $\mathbb{q}_{\gamma}(z) \prec \mathbb{q}(z), z \in U^{\circ}$. If $\Omega \neq \mathbb{C}$ is a simply connected domain, then $\Omega=k\left(U^{\circ}\right)$ considering a conformal mapping $k(z)$ of $U^{\circ}$ onto $\Omega$. In this case, the class $\theta_{\Gamma}\left[k\left(U^{\circ}\right), \mathbb{q}\right]$ can be written as $\theta_{\Gamma}[k, \mathbb{q}]$.

Now, we obtain the next two results from the above theorem and corollary.

Theorem 4. Assume that $Y \in \theta_{\Gamma}[k, \mathbb{q}]$, if $\mathbb{q} \in \mathfrak{Q}_{1}$ and $f \in \mho$ fulfills the condition (7) and

$$
\begin{gathered}
Y\left(\Gamma_{\pi, \rho, \beta, \mu} f(z), \Gamma_{\pi, \rho, \beta+1, \mu} f(z), \Gamma_{\pi, \rho, \beta+2, \mu} f(z), \Gamma_{\pi, \rho, \beta+3, \mu} f(z), \Gamma_{\pi, \rho, \beta+4, \mu} f(z) ; z\right) \prec k(z), \\
\text { then } \Gamma_{\pi, \rho, \beta, \mu} f(z) \prec \mathbb{q}(z), z \in U^{\circ} .
\end{gathered}
$$

Corollary 2. Assume the function $\mathbb{q}(z)$ is a univalent in $U^{\circ}, \mathbb{q}(0)=1$ and $\Omega \subset \mathbb{C}$. Assume $Y \in \theta_{\Gamma}\left[k, \mathbb{A}_{\gamma}\right]$ for several $\gamma \in(0,1)$ such that $\mathbb{A}_{\gamma}(z)=\mathbb{q}(\gamma z)$. If $\mathbb{A}_{\gamma}$ satisfies the condition (17), $f \in \mho$ and

$$
Y\left(\Gamma_{\pi, \rho, \beta, \mu} f(z), \Gamma_{\pi, \rho, \beta+1, \mu} f(z), \Gamma_{\pi, \rho, \beta+2, \mu} f(z), \Gamma_{\pi, \rho, \beta+3, \mu} f(z), \Gamma_{\pi, \rho, \beta+4, \mu} f(z) ; z\right) \prec k(z),
$$

then

$$
\Gamma_{\pi, \rho, \beta, \mu} f(z) \prec \mathbb{q}(z), z \in U^{\circ} .
$$

Now, the next theorem gives the best dominant of the differential subordination (18).

Theorem 5. Suppose $Y: \mathbb{C}^{5} \times U^{\circ} \rightarrow \mathbb{C}$; also assume the function $k$ is univalent in $U^{\circ}$, and the differential equation

$$
\begin{aligned}
& Y\left(\mathbb{q}(z), \frac{z_{q^{\prime}}(z)+\mu q(z)}{1+\mu}, \frac{z^{2} \mathbb{q}^{\prime \prime}(z)+(1+2 \mu) z_{\mathbb{q}^{\prime}}(z)+\mu^{2} \Phi(z)}{(1+\mu)^{2}},\right.
\end{aligned}
$$

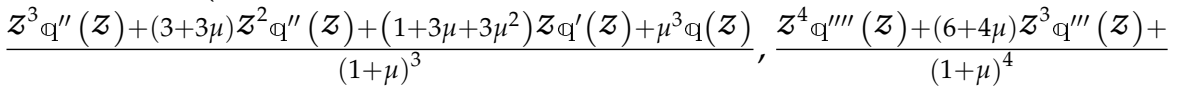

$$
\begin{aligned}
& \left.\frac{\left(7+12 \mu+4 \mu^{2}\right) \boldsymbol{z}^{2} \mathbb{q}^{\prime \prime}(\boldsymbol{z})+\left(1+4 \mu+4 \mu^{2}+4 \mu^{3}\right) \boldsymbol{Z}_{q^{\prime}}(\boldsymbol{z})+\mu^{4} \mathbb{q}(\boldsymbol{z}) ; \boldsymbol{z}}{(1+\mu)^{4}}\right)=k(\boldsymbol{z}),
\end{aligned}
$$

has a solution $\mathbb{q}(z)$ with $\mathbb{q}(0)=1$ and $\mathbb{q}(z)$ verifies Equation (7). If the function $f \in M$ satisfies condition (18) and

$$
Y\left(\Gamma_{\pi, \rho, \beta, \mu} f(z), \Gamma_{\pi, \rho, \beta+1, \mu} f(z), \Gamma_{\pi, \rho, \beta+2, \mu} f(z), \Gamma_{\pi, \rho, \beta+3, \mu} f(z), \Gamma_{\pi, \rho, \beta+4, \mu} f(z) ; z\right)
$$

is analytic in $U^{\circ}$, then

$\Gamma_{\pi, \rho, \beta, \mu} f(z) \prec \mathbb{q}(z)$, and $\mathbb{q}(z)$ is the best dominant. 
Proof. By applying Theorem 3, it can be shown that $\mathbb{q}(z)$ is a dominant of Equation (18), because $\mathbb{q}(\boldsymbol{z})$ satisfies (19), so that $\mathbb{q}(z)$ is a solution of $(18)$ and hence $\mathbb{q}(z)$ will be dominant of all dominants; therefore $\mathbb{q}(z)$ will be the best dominant.

Now, we put $\mathbb{q}(z)=\mathbb{M} z, \mathbb{M}>0$, and using Definition 7, the class of admissible functions $\theta_{\Gamma}[\Omega, \mathbb{q}]$, denoted by $\theta_{\Gamma}[\Omega, \mathbb{M}]$, is given below.

Definition 8. Assume that $\mathbb{M}>0$ and that $\Omega$ is a set in $\mathbb{C}$. The class of admissible functions $\theta_{\Gamma}[\Omega, \mathbb{M}]$ consists of those functions $Y: \mathbb{C}^{5} \times U^{\circ} \rightarrow \mathbb{C}$ that satisfy the admissibility condition:

$$
\begin{gathered}
\mathrm{Y}\left(\mathbb{M} e^{i \vartheta}, \frac{\mathbb{k}+\mu}{1+\mu} \mathbb{M} e^{i \vartheta}, \frac{\mathbb{L}+\left[(2 \mu+1) \mathbb{k}+\mu^{2}\right] \mathbb{M} e^{i \vartheta}}{(1+\mu)^{2}}, \frac{\mathbb{N}+(3 \mu+3) \mathbb{L}+\left[3 \mu^{2}+3 \mu+1\right) \mathbb{k}+\mu^{3}}{(1+\mu)^{3}},\right. \\
\left.\frac{\mathbb{A}+(4 \mu+6) \mathbb{N}+\left(4 \mu^{2}+12 \mu+7\right) \mathbb{L}+\left[\left(4 \mu^{3}+4 \mu^{2}+4 \mu+1\right) \mathbb{k}+\mu^{4}\right] \mathbb{M} e^{i \vartheta}}{(1+\mu)^{4}} ; z\right) \notin \Omega,
\end{gathered}
$$

such that $1>-\mu, z \in U^{\circ}, \mathcal{R} e\left(\mathbb{L} e^{-i \vartheta}\right) \geq(\mathbb{k}-1) \mathbb{k} \mathbb{M}, \mathcal{R} e\left(\mathbb{N} e^{-i \vartheta}\right) \geq 0$ and $\operatorname{Re}\left(\mathbb{A} e^{-i \vartheta}\right) \geq 0$ for all $\vartheta \in R$ and $\mathbb{k} \geq 3$.

Theorem 6. Assume that $Y \in \theta_{\Gamma}[\Omega, \mathbb{M}]$. If $f \in M$ fulfills the conditions: $\left|\Gamma_{\pi, \rho, \beta+2, u} f(z)\right| \leq$ $\mathbb{k}^{2} \mathbb{M}, \mathbb{k} \geq 3, \mathbb{M}>0$, and

$$
Y\left(\Gamma_{\pi, \rho, \beta, \mu} f(z), \Gamma_{\pi, \rho, \beta+1, \mu} f(z), \Gamma_{\pi, \rho, \beta+2, \mu} f(z), \Gamma_{\pi, \rho, \beta+3, \mu} f(z), \Gamma_{\pi, \rho, \beta+4, \mu} f(z) ; z\right) \in \Omega,
$$

then $\left|\Gamma_{\pi, \rho, \beta, \mu} f(z)\right|<\mathbb{M}$.

Now, taking $\Omega=\mathbb{q}\left(U^{\circ}\right)=\{w:|w|<M\}$, the class $\theta_{\Gamma}[\Omega, \mathbb{M}]$ is simply denoted by $\theta_{\Gamma}[\mathbb{M}]$.

Theorem 7. Assume $\mathbb{k} \geq 3, \mathbb{M}>0, \mu>-1$. If $f \in M$ satisfies the conditions $\left|\Gamma_{\pi, \rho, \beta+2, \mu} f(z)\right| \leq$ $\mathbb{k}^{2} \mathbb{M}$, and $\left|(1+\mu)^{4} \Gamma_{\pi, \rho, \beta+4, \mu} f(z)-\mu(1+\mu)^{3} \Gamma_{\pi, \rho, \beta+3, \mu} f(z)\right|<\left(\left|1+3 \mu+\delta \mu^{2}+\mu^{3}\right|+2 \mid 7+\right.$ $\left.9 \mu+\mu^{2} \mid\right) 3 \mathbb{M}$, then $\left|\Gamma_{\pi, \rho, \beta, \mu} f(z)\right|<\mathbb{M}$.

Proof. Assume that $\mathrm{Y}(\mathrm{r}, \mathbb{\mathrm { S }}, \mathbb{\mathrm { x }}, \mathrm{y}, \mathrm{g}, z)=(1+\mu)^{4} \mathrm{~g}-\mu(1+\mu)^{3} \mathrm{y}, \Omega=k\left(U^{\circ}\right)$, such that

$$
k(z)=\left(\left|1+3 \mu+\mu^{2}+\mu^{3}\right|+2\left|7+9 \mu+\delta \mu^{2}\right|\right) 3 \mathbb{M} z, \mathbb{M}>0 .
$$

Now, by applying Theorem 6 , we show that $Y \in \theta_{\Gamma, 1}[\Omega, \mathbb{M}]$. Because

$$
\begin{gathered}
\mid \mathrm{Y}\left(\mathbb{M} e^{i \vartheta}, \frac{\mathbb{k}+\mu}{1+\mu} \mathbb{M} e^{i \vartheta}, \frac{\mathbb{L}+\left[(2 \mu+1) \mathbb{k}+\mu^{2}\right] \mathbb{M} e^{i \vartheta}}{(1+\mu)^{2}}, \frac{\mathbb{N}+(3+3 \mu) \mathbb{L}+\left[1+3 \mu+3 \mu^{2}\right) \mathbb{k}+\mu^{3}}{(1+\mu)^{3}},\right. \\
\left.\frac{\mathbb{A}+(6+4 \mu) \mathbb{N}+\left(7+12 \mu+4 \mu^{2}\right) \mathbb{L}+\left[\left(1+4 \mu+4 \mu^{2}+4 \mu^{3}\right) \mathbb{k}+\mu^{4}\right] \mathbb{M} e^{i \vartheta}}{(1+\mu)^{4}} ; z\right) \mid \\
=\left|\mathbb{A}+(6+3 \mu) \mathbb{N}+\left(7+9 \mu+\mu^{2}\right) \mathbb{L}+\left(1+3 \mu+\mu^{2}+\mu^{3}\right) \mathbb{k} \mathbb{M} e^{i \vartheta}\right| \\
=\left|\mathbb{A} e^{-i \vartheta}+(6+3 \mu) \mathbb{N} e^{-i \vartheta}+\left(7+9 \mu+\mu^{2}\right) \mathbb{L} e^{-i \vartheta}+\left(1+3 \mu+\mu^{3}\right) \mathbb{k} \mathbb{M}\right| \\
\geq \mathcal{R} e\left(\mathbb{A} e^{-i \vartheta}\right)+|(6+3 \mu)| \mathcal{R} e\left(\mathbb{N} e^{-i \vartheta}\right)+\left|\left(7+9 \mu+\mu^{2}\right)\right| \mathbb{L} e^{-i \vartheta}+\left|\left(1+3 \mu+\mu^{2}+\mu^{3}\right)\right| \mathbb{k} \mathbb{M}, \\
\geq\left|\left(1+3 \mu+\mu^{2}+\mu^{3}\right)\right| \mathbb{k} \mathbb{M}+2\left|\left(7+9 \mu+\mu^{2}\right)\right| \mathbb{k}(\mathbb{k}-1) \mathbb{M} \\
\geq\left(\left|\left(1+3 \mu+\mu^{2}+\mu^{3}\right)\right|+2\left|\left(7+9 \mu+\mu^{2}\right)\right|\right) 3 \mathbb{M},
\end{gathered}
$$

such that

$$
\begin{gathered}
\mathcal{R} e\left(\mathbb{A} e^{-i \vartheta}\right) \geq 0, \mathcal{R} e\left(\mathbb{N} e^{-i \vartheta}\right) \geq 0 \text { and } \mathcal{R} e\left(\mathbb{L} e^{-i \vartheta}\right) \geq(\mathbb{k}-1) \mathbb{k} \mathbb{M} a \\
\text { for all } \vartheta \in R, z \in U^{\circ} \text { and } \mathbb{k} \geq 3 .
\end{gathered}
$$

The proof is complete. 


\section{Fourth-Order Differential Superordination Results Using the Operator $\Gamma_{\pi, \rho, \beta, \mu} f(z)$}

In this section, we introduce fourth-order differential superordination by using $\Gamma_{\pi, \rho, \beta, \mu} f(z)$ defined by (2). For this main aim, the class of admissible functions is given by the definition below:

Definition 9. Assume $\mathfrak{q}^{\prime}(\boldsymbol{z}) \neq 0, \mathbb{q} \in \mathcal{K}_{1}$ and let $\Omega$ be a set in $\mathbb{C}$. The admissible class $Y_{\Gamma}^{\prime}[\Omega, \mathbb{q}]$ consists of those functions $Y: \mathbb{C}^{5} \times \bar{U}^{\circ} \rightarrow \mathbb{C}$ that satisfy the admissibility condition

$$
Y(\mathbb{r}, \mathbb{s}, \mathbb{x}, \mathbb{y}, \mathrm{g} ; \mathcal{J}) \in \Omega,
$$

where

$$
\begin{aligned}
& \mathbb{r}=\mathbb{q}(z), \mathbb{\$}=\frac{z \mathcal{J} \mathbb{q}^{\prime}(z)+\mathfrak{m} \mathbb{q}(z)}{(1+\mu) \mathfrak{m}}, \\
& \mathcal{R} e\left\{\frac{(1+\mu)^{2} \mathbb{x}-\mu^{2} \mathrm{r}}{(1+\mu) \mathbb{s}-\mu \mathrm{r}}-2 \mu\right\} \geq \frac{1}{\mathfrak{m}} \mathcal{R e}\left\{\frac{z_{\mathbb{q}^{\prime \prime}}(z)}{\mathbb{q}^{\prime}(z)}+1\right\}, \\
& \mathcal{R} e\left\{\frac{(1+\mu)^{2}[(1+\mu) \mathrm{y}-(3+3 \mu) \mathbb{x}]+\left(3 \mu^{2}+2 \mu^{3}\right) \mathrm{r}}{(1+\mu) \mathbb{s}-\mu \mathrm{r}}+\left(2+6 \mu+3 \mu^{2}\right)\right\} \geq \frac{1}{\mathfrak{m}^{2}} \mathcal{R} e\left\{\frac{z^{2} \mathbb{q}^{\prime \prime \prime}(z)}{\mathbb{q}^{\prime}(\boldsymbol{z})}\right\},
\end{aligned}
$$

and

$$
\begin{aligned}
\mathcal{R} e\{ & \frac{(1+\mu)\left[(1+\mu)^{3} \mathrm{~g}-(1+\mu)^{2}(6+4 \mu) \mathrm{y}+(1+\mu)\left(11+18 \mu+8 \mu^{2}\right) \mathbb{x}\right.}{(1+\mu) \mathbb{s}+\mu \mathrm{r}} \\
& \left.\frac{\left.-\left(6+22 \mu+18 \mu^{2}+8 \mu^{3}\right) \mathbb{s}\right]+\left(6 \mu+11 \mu^{2}+6 \mu^{3}+3 \mu^{4}\right) \mathrm{r}}{(1+\mu) \mathbb{s}+\mu \mathrm{r}}\right\} \geq \frac{1}{\mathfrak{m}^{3}} \mathcal{R e}\left\{\frac{\boldsymbol{Z}^{3} \mathbb{q}^{\prime \prime \prime \prime}(\boldsymbol{z})}{\mathfrak{q}^{\prime}(\boldsymbol{Z})}\right\},
\end{aligned}
$$

where $z \in U^{\circ}, \mathcal{J} \in \partial U^{\circ}, \mu \in \mathbb{C} \backslash Z_{O}^{-}, Z_{O}^{-}=\{0,-1,-2, \ldots\}$ and $\mathfrak{m} \geq 3$.

Theorem 8. Assume that $Y \in \theta_{\Gamma}^{\prime}[\Omega, \mathbb{q}]$. If $f \in M$ and $\Gamma_{\pi, p, \beta, \mu} f(z) \in \mathbb{Q}_{1}$ satisfy the conditions

$$
\mathcal{R} e\left(\frac{z^{2} \mathbb{q}^{\prime \prime \prime}(z)}{\mathbb{q}^{\prime}(\boldsymbol{z})}\right) \geq 0, \quad\left|\left(\frac{\Gamma_{\pi, \rho, \beta, \mu} f(z)}{\mathbb{q}^{\prime}(z)}\right)\right| \leq \frac{1}{\mathfrak{m}^{2}}
$$

and

$$
\left\{\mathrm{Y}\left(\Gamma_{\pi, \rho, \beta, \mu} f(z), \Gamma_{\pi, \rho, \beta+1, \mu} f(z), \Gamma_{\pi, \rho, \beta+2, \mu} f(z), \Gamma_{\pi, \rho, \beta+3, \mu} f(z), \Gamma_{\pi, \rho, \beta+4, \mu} f(z)\right): z \in U^{\circ}\right\}
$$

is univalent in $U^{\circ}$, and

$$
\begin{gathered}
\Omega \subset\left\{\mathrm{Y}\left(\Gamma_{\pi, \rho, \beta, \mu} f(z), \Gamma_{\pi, \rho, \beta+1, \mu} f(z), \Gamma_{\pi, \rho, \beta+2, \mu} f(z), \Gamma_{\pi, \rho, \beta+3, \mu} f(z), \Gamma_{\pi, \rho, \beta+4, \mu} f(z) ; z \in U^{\circ}\right)\right\}, \\
\text { then } \mathbb{q}(z) \prec \Gamma_{\pi, \rho, \beta, \mu} f(z) .
\end{gathered}
$$

Proof. Define the functions $\mathrm{p}(\boldsymbol{z})$ and $\psi$ by (9) and (15), respectively. We have $Y \in \theta_{\Gamma}^{\prime}[\Omega, \mathbb{q}]$. Therefore, from (16) and (22), we obtain

$$
\Omega \subset\left\{\psi\left(p(z), z p^{\prime}(z), z^{2} p^{\prime \prime}(z), z^{3} p^{\prime \prime \prime}(z), z^{4} p^{\prime \prime \prime \prime}(z) ; z \in U^{\circ}\right)\right\} .
$$

Now, from Equation (14), note that the admissibility condition for $Y \in \theta_{\Gamma}^{\prime}[\Omega, \mathbb{q}]$ in Definition 9 is the admissiblity condition for $\psi$ as defined in Definition 6 with $\mathfrak{n}=3$.

Therefore, by applying (7) and Theorem 2 and knowing $\psi \in \theta_{\Gamma}^{\prime}[\Omega, \mathbb{q}]$, we obtain $\mathbb{q}(z) \prec \mathrm{p}(z)=\Gamma_{\pi, \rho, \beta, \mu} f(z)$.

Hence, the proof of theorem is complete.

Now, if $\Omega=k\left(U^{\circ}\right)$ for a conformal mapping $k(z)$ of $U^{\circ}$ onto $\Omega$ and if $\Omega \neq \mathbb{C}$ is a simply connected domain then the class $\theta_{\Gamma}^{\prime}\left[k\left(U^{\circ}\right), \mathbb{q}\right]$ is written as $\theta_{\Gamma}^{\prime}[k, \mathbb{q}]$.

The below theorem is direct consequence of the theorem above. 
Theorem 9. Consider the analytic function $k(z)$ in $U^{\circ}$ and $Y \in \theta_{\Gamma}^{\prime}\left[k\left(U^{\circ}\right)\right.$, $\left.\mathbb{q}\right]$. If $f \in$ $M, \Gamma_{\pi, \rho, \beta, \mu} f(z) \in \mathbb{Q}_{1}$ and $\mathbb{q} \in \mathcal{K}_{1}$ satisfies the condition (21),

$$
\left\{Y\left(\Gamma_{\pi, \rho, \beta, \mu} f(z), \Gamma_{\pi, \rho, \beta+1, \mu} f(z), \Gamma_{\pi, \rho, \beta+2, \mu} f(z), \Gamma_{\pi, \rho, \beta+3, \mu} f(z), \Gamma_{\pi, \rho, \beta+4, \mu} f(z)\right): z \in U^{\circ}\right\}
$$

is univalent in $U^{\circ}$, and

$$
\begin{gathered}
k(z) \subset\left\{Y\left(\Gamma_{\pi, \rho, \beta, \mu} f(z), \Gamma_{\pi, \rho, \beta+1, \mu} f(z), \Gamma_{\pi, \rho, \beta+2, \mu} f(z), \Gamma_{\pi, \rho, \beta+3, \mu} f(z), \Gamma_{\pi, \rho, \beta+4, \mu} f(z) ; z \in U^{\circ}\right)\right\}, \\
\text { then } \mathbb{q}(z) \prec \Gamma_{\pi, \rho, \beta, \mu} f(z) .
\end{gathered}
$$

Proof. The proof of theorem is similar to that of Theorem 3 and is omitted here.

Theorem 10. Assume that $Y: \mathbb{C}^{5} \times \bar{U}^{\circ} \rightarrow \mathbb{C}$, the function $k(z)$ is analytic in $U^{\circ}$, and $\psi$ is defined by (15). Assume that the differential equation

$$
\left\{\psi\left(p(z), z p^{\prime}(z), z^{2} p^{\prime \prime}(z), z^{3} p^{\prime \prime \prime}(z), z^{4} p^{\prime \prime \prime \prime}(z) ; z \in U^{\circ}\right)\right\}=k(z),
$$

has a solution $\mathbb{q}(\boldsymbol{z}) \in \mathbb{Q}_{1}$. If $\Gamma_{\pi, \rho, \beta, \mu} f(\boldsymbol{z}) \in \mathbb{Q}_{1}, \mathbb{q} \in \mathcal{K}_{1}, \mathbb{q}^{\prime}(\boldsymbol{z}) \neq 0$ and $f \in M$ satisfy the conditions (7) and (21),

$$
\left\{Y\left(\Gamma_{\pi, \rho, \beta, \mu} f(z), \Gamma_{\pi, \rho, \beta+1, \mu} f(z), \Gamma_{\pi, \rho, \beta+2, \mu} f(z), \Gamma_{\pi, \rho, \beta+3, \mu} f(z), \Gamma_{\pi, \rho, \beta+4, \mu} f(z)\right): z \in U^{\circ}\right\}
$$

is univalent in $U^{\circ}$, and

$$
k(z) \subset\left\{Y\left(\Gamma_{\pi, \rho, \beta, \mu} f(z), \Gamma_{\pi, \rho, \beta+1, \mu} f(z), \Gamma_{\pi, \rho, \beta+2, \mu} f(z), \Gamma_{\pi, \rho, \beta+3, \mu} f(z), \Gamma_{\pi, \rho, \beta+4, \mu} f(z) ; z \in U^{\circ}\right)\right\},
$$

then $\mathbb{q}(z) \prec \Gamma_{\pi, \rho, \beta, \delta} f(z)$, and $\mathbb{q}(z)$ is the best subordinate of (23).

Proof. The proof of theorem is similar to that of Theorem 5 and is omitted here.

\section{Sandwich-Type Results}

Now, by using Theorems 5 and 9, we have the sandwich-type result.

Theorem 11. Consider two analytic functions $k_{1}(z)$ and $\mathbb{q}_{1}(z)$ in $U^{\circ}$, and $\mathbb{1}_{2}(z) \in \mathbb{Q}_{1}$ with $\mathbb{q}_{1}(0)=\mathbb{q}_{2}(0)=1$. In addition let the function $k_{2}(z)$ be univalent in $U^{\circ}$ and $Y \in$ $\theta_{\Gamma}\left[k_{2}, \mathbb{Q}_{2}\right] \cap \theta_{\Gamma}^{\prime}\left[k_{1}, \mathbb{Q}_{1}\right]$. If $\Gamma_{\pi, \rho, \beta, \mu} f(z) \in \mathbb{Q}_{1} \cap \mathcal{K}, f \in M$,

$$
\left\{Y\left(\Gamma_{\pi, \rho, \beta, \mu} f(z), \Gamma_{\pi, \rho, \beta+1, \mu} f(z), \Gamma_{\pi, \rho, \beta+2, \mu} f(z), \Gamma_{\pi, \rho, \beta+3, \mu} f(z), \Gamma_{\pi, \rho, \beta+4, \mu} f(z)\right): z \in U^{\circ}\right\}
$$

is univalent in $U^{\circ}$, and the two conditions (7) and (21) are satisfied as

$$
\begin{gathered}
k_{1}(z) \prec\left\{Y\left(\Gamma_{\pi, \rho, \beta, \mu} f(z), \Gamma_{\pi, \rho, \beta+1, \mu} f(z), \Gamma_{\pi, \rho, \beta+2, \mu} f(z), \Gamma_{\pi, \rho, \beta+3, \mu} f(z), \Gamma_{\pi, \rho, \beta+4, \mu} f(z) ; z \in U^{\circ}\right)\right\} \prec k_{2}(z), \\
\text { then } \\
\mathbb{Q}_{1}(z) \prec \Gamma_{\pi, \rho, \beta, \mu} f(z) \prec \mathbb{q}_{2}(z)
\end{gathered}
$$

\section{Conclusions}

A new differential operator is introduced in the present paper in Definition 1. Using the concepts of fourth-order differential subordination and superordination, the classes of admissible functions are defined related to each of the two concepts, and using those definitions, several theorems are proved involving the newly defined operator regarding fourth-order subordinations in Section 3 and regarding fourth-order superordination in Section 4. By applying a well-known technique, a sandwich-type theorem is stated in 
Section 5 of the paper combining the subordination and superordination results obtained before. The results presented here could inspire future work involving other operators for obtaining fourth-order differential subordinations and superordinations. Certain special classes of univalent functions could be introduced using the operator defined in this paper, and studies for obtaining properties of those classes could be done invoking the notions of fourth-order differential subordination and superordination using the admissibility conditions given here in Definition 7, Definition 8 and Definition 9 and the best dominant obtained in Theorem 5 .

Author Contributions: Conceptualization, methodology, software by A.A.L., validation, formal analysis, investigation, resources, by S.S.A., data curation, writing-original draft preparation, writing-review and editing, visualization by W.G.A., supervision, project administration, funding acquisition, by B.K.M. All authors have read and agreed to the published version of the manuscript.

Funding: This research received no external funding.

Institutional Review Board Statement: Not applicable.

Informed Consent Statement: Not applicable.

Data Availability Statement: Not applicable.

Conflicts of Interest: The authors declare no conflict of interest.

\section{References}

1. Ali, R.M.; Ravichandran, V.; Seenivasagan, N. Differential subordination and superordination of analytic functions defined by multiplier transformation. Math. Inequal. Appl. 2009, 12, 123-139. [CrossRef]

2. Al-Ameedee, S.A.; Atshan, W.G.; Ali AL-Maamori, F. On sandwich results of univalent functions defined by a linear operator. J. Interdiscip. Math. 2020, 23, 803-809. [CrossRef]

3. Al-Ameedee, S.A.; Atshan, W.G.; Ali AL-Maamori, F. Some new results of differential subordinations for higher-order derivatives of multivalent functions. J. Phys. Conf. Ser. 2021, 1804, 012111. [CrossRef]

4. Antonino, J.A.; Miller, S.S. Third-order differential inqualities and subordination in the complex plane. Complex Var. Ellptic Equ. 2011, 56, 439-454. [CrossRef]

5. Aouf, M.K.; Seoudy, T.M. Subordination and superordination of a certain integral operator on meromorphic functions. Comput. Math. Appl. 2010, 59, 3669-3678. [CrossRef]

6. Aouf, M.K.; Seoudy, T.M. Certain Class of Bi-Bazilevic Functions with Bounded Boundary Rotation Involving Salăgeăn Operator. Constr. Math. Anal. 2020, 3, 139-149. [CrossRef]

7. Atshan, W.G.; Ali, A.A.R. On some sandwich theorems of analytic functions involving Noor-Sălăgean operator. Adv. Math. Sci. J. 2020, 9, 8455-8467. [CrossRef]

8. Atshan, W.G.; Ali, A.A.R. On sandwich theorems results for certain univalent functions defined by generalized operators. Iraqi J. Sci. 2021, 62, 2376-2383. [CrossRef]

9. Atshan, W.G.; Abbas, I.A.; Yalcin, S. New concept on Fourth-order differential subordination and superordination with some results for multivalent analytic functions. J. Al-Qadisiyah Comput. Sci. Math. 2020, 12, 96-107.

10. Atshan, W.G.; Battor, A.H.; Abaas, A.F. On third-order differential subordination results for univalent analytic functions involving an operator. J. Phys. Conf. Ser. 2020, 1664, 012041. [CrossRef]

11. Atshan, W.G.; Battor, A.H.; Abaas, A.F. Some sandwich theorems for meromorphic univalent functions defined by new integral operator. J. Interdiscip. Math. 2021, 24, 579-591. [CrossRef]

12. Atshan, W.G.; Battor, A.H.; Abaas, A.F.; Oros, G.I. New and extended results on fourth-order differential subordination for univalent analytic functions. Al-Qadisiyah J. Pure Sci. 2020, 25, 1-13. [CrossRef]

13. Atshan, W.G.; Hadi, R.A. Some differential subordination and superordination results of p-valent functions defined by differential operator. J. Phys. Conf. Ser. 2020, 1664, 012043. [CrossRef]

14. Atshan, W.G.; Hassan, H.Z.; Yalcin, S. On third-order differential subordination results for univalent functions defined by differential operator. Uzb. Math. J. 2021, 62, 26-42.

15. Atshan, W.G.; Kulkarni, S.R. On application of differential subordination for certain subclass of meromorphically p-valent functions with positive coefficients defined by linear operator. J. Inequalities Pure Appl. Math. 2009, 10, 11.

16. Attiya, A.A.; Kwon, O.S.; Hang, P.J.; Cho, N.E. An Integrodifferential operator for meromorphic functions associated with the Hurwitz-Lerch Zeta function. Filomat 2016, 30, 2045-2057. [CrossRef]

17. NCho, E.; Kim, T.H. Multiplier transformations and strongly close-to convex functions. Bull. Korean Math. Soc. 2003, 40, 399-410.

18. Cotîrlă, L.I.; Cătaş, A. Differential sandwich theorem for certain class of analytic functions associated with an integral operator. Studia Univ. Babes-Bolyai Math. 2020, 65, 487-494. [CrossRef] 
19. Kavitha, S.; Sivasubramanian, S.; Jayasankar, R. Differential sudordination and superordination results for Cho-Kwon- Srivastava operator. Comput. Math. Appl. 2012, 64, 1789-1803. [CrossRef]

20. Miller, S.S.; Mocanu, P.T. Differential Subordinations: Theory and Applications; Series on Monographs and Textbook in Pure and Applied Mathematics, No. 225; Marcel Dekker Inc.: New York, NY, USA; Besel, The Netherlands, 2000.

21. Miller, S.S.; Mocanu, P.T. Subordinants of differential superorordinations. Complex Var. Theory Appl. 2003, 48, 815-826.

22. Oros, G.I. Study on new integral operators defined using confluent hypergeometric function. Adv. Differ. Equ. 2021, 2021, 342. [CrossRef]

23. Ponnusamy, S.; Juneja, O.P. Third-order differential inequalities in the complex plane. In Current Topics in Analytic Function Theory; World Scientific Publising Company: Singapore; Hackensack, NJ, USA; London, UK; Hongkong, China, 1992.

24. Shanmugam, T.N.; Sivasubramanian, S.; Srivastava, H.M. Differential sandwich theorems for certain subclasses of analytic functions involving multiplier transformatios. Integral Transform. Spec. Funct. 2006, 17, 889-899. [CrossRef]

25. Wanas, A.K.; Cotîrlă, L.I. Initial Coefficient Estimates, Fekete-Szegö Inequalities for New Families of Bi-Univalent Functions Governed by (p- q)-Wanas Operator. Symmetry 2021, 13, 2118. [CrossRef]

26. Farzana, H.A.; Stephen, B.A.; Jeyaraman, M.P. Third-order differential subordination of analytic function defined by functional derivative operator. Ann. Alexandru Ioan Cuza Univ. -Math. 2016, 62, 105-120.

27. Cotirlă, L. A differential sandwich theorem for analytic functions defined by the integral operator. Stud. Univ. Babes-Bolyal Math $2009,54,13-21$.

28. Murugusundaramoorthy, G.; Magesh, N. An application of second order differential inequalities based on linear and integral operators. Int. J. Math. Sci. Eng. Appl. 2008, 2, 105-114.

29. Raducanu, D. Third order differential subordinations for analytic functions associated with generalized Mittag-Leffler functions. Mediterr. J. Math. 2017, 14, 1-18. [CrossRef]

30. Tang, H.; Srivastava, H.M.; Deniz, E.; Li, S. Third-order differential superordination involving the generalized Bessel functions. Bull. Malays. Math. Sci. Soc. 2015, 38, 1669-1688. [CrossRef]

31. Tang, H.; Srivastava, H.M.; Li, S.; Ma, L. Third-order differential subordination and superordination results for meromorphically multivalent functions associated with the Liu-Srivastava operator. Abstract Appl. Anal. 2014, 2014, 11. [CrossRef]

32. Tang, H.; Deniz, E. Third-order differential subordination results for analytic functions involving the generalized Bessel functions. Acta Math. Sci. 2014, 34, 1707-1719. [CrossRef]

33. Gochhayat, P. Sandwich-type results for a class of functions defined by a generalized differential operator. Math. Vesink 2013, 65, 178-186.

34. Gochhayat, P.; Prajapati, A. Applications of third order di_erential subordination and superordination involving generalized Struve function. Filomat 2019, 33, 3047-3059. [CrossRef]

35. El-Ashwah, R.M.; Aouf, M.K. Differential subordination and superordination for certain subclasses of p-valent functions. Math Comput. Model. 2010, 51, 349-360. [CrossRef] 